and where the light approaches in two regular streams of equal concentration. The difference is due to the position of the opening $\mathrm{BC}$ relative to collision centres below it within less than free path distances, from which the approaching molecules will start, with equal probability of movement in all directions. The molecular problem would thus appear to approximate to the simple statement in my paper, in which the "points $\mathrm{O}$ " may consequently be regarded as little elements of area, and to be quite different from light calculations such as the one worked out above.

In connexion with Mr. Atkinson's claim that elementary principles would be defied if an effect were to occur, it is not obvious why this should be so (provided, as has always been emphasised, that the apparatus shall be sufficiently small to deal with the oscillations or movements of molecules individually), any more than in the case of the energy of a swinging pendulum being converted into useful external work by the agency of suitable mechanism.

The argument that if light is reflected, then molecules must actually be so similarly, to which Mr. Atkinson strongly objects, does not appear in my paper, where, in fact, the exact opposite is stated (page I053, line 26), although such reflection, as an average effect, is not regarded as being impossible.

King's College, University of London, Strand, W.C.2, June 22.

Polarisation of Diffused Light under the Sea.

I Took advantage of a recent opportunity to make some observations on polarisation of the diffused light in sea-water, using a detector consisting of four quartz prisms made up on the De Senarmont principle, combined with a Nicol. The depth was 3o feet, the sea-water very clear, and the day cloudy, with no trace of sky polarisation. The diffused light at the bottom was quite strongly polarised, the water behaving like a turbid medium observed at right angles to the incident beam, the plane of polarisation being perpendicular to the surface.

The greatest intensity was in the horizontal direction, diminishing rapidly as the angle of elevation increased, and disappearing completely long before the direction became nearly vertical. Repeated observations created a strong impression that the direction of maximum polarisation was not exactly horizontal, but very slightly inclined downwards. However, the difference, if any, was so small that it must be regarded as doubtful.

I should have liked to repeat observations on a day of blue sky, but the opportunity did not arise. The light from the sandy bottom and from a white plate did not show any trace of polarisation.

Apart from polarisation, it was interesting to observe the surface. It is not easy to look vertically upwards in a diver's helmet, but there was evidently a circular luminous area directly overhead, rapidly falling off in intensity without any sharpness of transition. It was a kind of inverse penumbra effect. E. E. Brooks

Leicester City Technical School, June 30, I922.

\section{Discoveries in Tropical Medicine.}

In NATURE of June 24 Sir Ray Lankester repeats his statement that the transmission of Filaria bancrofti from infected to healthy men through the intermediation of the mosquito is not a sufficiently established fact. I trust you will permit me to state, for the benefit of those of your readers who may be puzzled by an assertion so discordant with current teaching, that at this school we shall always be happy to demonstrate sections of mosquitoes and of human lymphatic gland that show the facts of that transmission.

With regard to Sir Ray Lankester's other emphasised statement that "Manson did not discover the part played by the mosquito " in this transmission, we shall be happy to show Manson's original charts and drawings made in Amoy, and other necessary evidence that Manson did follow out the development of the embryonic and larval Filaria bancrofti in the stomach and body-cavity of the mosquito. This evidence, quite apart from any additions to it or corrected inferences from it, establishes the essential fact that the insect is the vital agent of transmission, since it releases the imprisoned embryo from the blood-vessels of its host, nourishes it until certain necessary organs are developed, and thus enables it to make a start in life.

London School of Tropical Medicine

Endsleigh Gardens, Euston Road, N.W.r, July 5 .

\section{Ouramoba.}

WiTH regard to the notes by Messrs. Rowley and Kirkpatrick in NATURE of July 8, on the occurrence of Leidy's genus Ouramœba in England, it may 'be of interest to record that I have recently (a few days before their letters were published) found one specimen of Amoeba proteus in this condition, i.e. infected with a parasitic alga. I was very much interested, because I have never encountered anything like Ouramoba in the neighbourhood of Manchester, where I have chiefly collected.

I have not Leidy's book with me now for reference but am familiar with his figures, and have no doubt whatever that the specimen which I found corresponds exactly with his genus Ouramœba. It was a typical Amoeba proteus, with filaments of the parasitic alga projecting fanwise in two tufts, one tuft on each side, nearer the end which was posterior in progression. Its vitality certainly was not impaired in any way.

This specimen was taken from a small tarn near Crag House farm, not far from Windermere. The tarn lies at the summit of a ridge, the altitude of which is given as 700 feet on the Ordnance Survey map. It should be emphasised that, although the tarn contains ordinary Amcba proteus in fair abundance, only one specimen has so far been seen which was infected with the alga. The other fauna of the tarn include very numerous Thecamcbida, some Flagellata, Ciliata, desmids, diatoms, etc., a fauna which corresponds fairly closely with that dealt with by Leidy in his book, and which is probably typical of open moorland country at high altitudes.

Amoba proteus is found in other tarns in this neighbourhood; but I have never seen any other specimen infected with the alga from these other localities, although, since reading Mr. Rowley's letter, I have again searched fresh material.

Bowness-on-Windermere, July Io.

$$
\text { G. I.APAGE. }
$$

\section{Histological Stains.}

WiTH reference to Dr. Nierenstein's remark (July 8 , p. 33) that the British dye industry would do well to pay attention to the supply of dyes suitable for histological work, it should be recorded that in I9I9, when pathologists here had a difficulty in getting satisfactory stains, the Pathological Society of Great Britain and Ireland approached Dr. Levinstein in the matter and received from his organisation ample and most useful help which carried us on until reasonably good stains became available again through the ordinary trade channels.

July 8.

NO. $275 \mathrm{I}$, VOL. I IO] 